\title{
Editorial About: "A Prospective, Open-Label, Multicenter Phase II Trial of Neoadjuvant Therapy Using Full-Dose Gemcitabine and S-1 Concurrent with Radiation for Resectable Pancreatic Ductal Adenocarcinoma"
}

\author{
Patrick B. Schwartz, $\mathrm{MD}^{1}$, Nataliya V. Uboha, $\mathrm{MD}, \mathrm{PhD}^{2}$, and Sharon M. Weber, $\mathrm{MD}^{1}$ \\ ${ }^{1}$ Department of Surgery, University of Wisconsin School of Medicine and Public Health, Madison, WI; ${ }^{2}$ Department of \\ Oncology, University of Wisconsin School of Medicine and Public Health, Madison, WI
}

Pancreatic ductal adenocarcinoma (PDAC) is a devastating disease that affected approximately 53,000 new patients in 2016. Patients with a diagnosis of this malignancy have a 5 -year overall survival rate of $10 \%$, which increases only to $25 \%$ for those with resectable disease. ${ }^{1,2}$ These poor outcomes reflect two notions. First, PDAC is a systemic disease at the time of diagnosis regardless of clinical stage. As such, treatment must include systemic therapy as one component of the therapy. Second, despite progressive advances during the last several years, current treatment options have limited efficacy at reducing recurrences and disease progression.

Historically, results from studies evaluating the use of radiotherapy for PDAC have been mixed, with adjuvant radiotherapy trials showing disparate results. ${ }^{3,4}$ The report entitled "A Prospective, Open-Label Multicenter Phase II Trial of Neoadjuvant Therapy Using Full-Dose Gemcitabine and S-1 Concurrent With Radiation for Resectable Pancreatic Ductal Adenocarcinoma" described the role of neoadjuvant radiation (total dose, $50.4 \mathrm{~Gy}$ ) given concurrently with full doses of chemotherapy (gemcitabine and S1). ${ }^{5}$ The authors demonstrated an impressive median survival of 55 months for 63 patients who had resectable and borderline resectable tumors treated with this regimen. ${ }^{5}$ This trial adds to the body of

(C) Society of Surgical Oncology 2019

First Received: 31 July 2019;

Published Online: 22 August 2019

S. M. Weber, MD

e-mail: webers@surgery.wisc.edu knowledge regarding the use of neoadjuvant chemoradiation in the treatment of pancreatic cancer. The unique aspect of this trial was full doses of chemotherapy delivered concurrently with radiation, thus targeting both local and distant disease.

The results of this study should be taken in light of other recent trials. The PREOPANC- 1 trial was a phase 3 trial that evaluated the role of preoperative chemoradiotherapy versus immediate surgery for 246 patients with resectable or borderline resectable tumors. ${ }^{6}$ All the patients received 6 months of perioperative gemcitabine. Despite equal total doses of systemic chemotherapy, the trial demonstrated improvements in both median recurrencefree survival [median, 9.9 vs 7.7 months; hazard ratio (HR), $0.67 ; p=0.010$ ] and overall survival (median, 17.1 vs 13.5 months; $\mathrm{HR}, 0.71 ; p=0.047$ ) with the neoadjuvant chemoradiation approach. It is important to note that the patients in the chemoradiation arm of the study received $30 \%$ of all the systemic chemotherapy during the preoperative period. Moreover, this trial did not use the more active systemic regimens available currently.

The randomized phase 2 and 3 Prep-02/JSAP-05 trial, which used the same chemotherapy regimen in the experimental arm as the current study used, compared the use of neoadjuvant chemotherapy with the use of upfront surgery followed by adjuvant S-1 chemotherapy. ${ }^{7}$ The study demonstrated a significantly improved median overall survival of 36.7 months in the neoadjuvant therapy cohort versus 26.7 months in the upfront surgery cohort $(p=0.015)$, with a 2-year overall survival rate of $64 \%$ versus 53\%. Therefore, the single-arm design of the current study begs the question regarding the individual 
contribution from chemotherapy and radiation. The strategy used in the current study demonstrated a prolonged median recurrence-free survival of 22.1 months, a median overall survival of 55.3 months, and overall survival rates of $76.7 \%$ at 1 year, $38.3 \%$ at 3 years, and $35.1 \%$ at 5-years. ${ }^{5}$

Other phase 2 trials using more active chemotherapy agents have shown better results than historical cohorts, but the single-arm design again precludes firm conclusions and changes to the standard of care. ${ }^{8}$ Until a randomized study is performed, it is not possible to decipher how much the radiation contributes when a more active chemotherapy backbone is used.

The aforementioned studies illuminate the difficulty of interpreting data from single-arm studies. Patient selection has the potential to influence survival outcomes by altering both disease biology and patient-related comorbidities. Notably, in the current study, only a single patient reportedly had borderline resectable disease, as defined by the National Cancer Care Network (NCCN), with the remaining patients defined as resectable. ${ }^{5}$ The patient with borderline resectable disease required a distal pancreatectomy with celiac axis resection. However, this is a rare operation, performed for only $2.4 \%$ of distal pancreatectomies. ${ }^{9}$ Despite this, 30\% (16/54) of the patients undergoing resection in this study required en bloc venous (portal/superior mesenteric vein) resection..$^{5}$ This seems to be discordant with the initial diagnosis of resectable disease if patients did not have progression. Therefore, without a clear idea of the patients' resectability status, generalization of the data is difficult without a comparative arm.

Given those caveats, with the paucity of prospective data from randomized studies that support neoadjuvant therapies for PDAC, the authors should be commended for completion of this trial. However, an immense opportunity remains to pursue further understanding of disease biology in prospective trials and to evaluate the role of neoadjuvant radiotherapy in combination with current standard chemotherapy in a randomized manner. The eagerly awaited results from Alliance for Clinical Trials in Oncology (ALLIANCE) Trial A021501 will provide answers this question. ${ }^{10}$ The trial compares neoadjuvant FOLFIRINOX alone and FOLRINOX followed by stereotactic body radiation for patients with borderline resectable PDAC.

Until further prospective evaluation is obtained, some insight can be gleaned from retrospective cohort studies. In a study evaluating approximately 260 patients who received either neoadjuvant chemoradiotherapy or chemotherapy alone, propensity-scored analysis showed increased local control with decreased positive margins and lymph nodes but no change in overall survival for the chemoradiotherapy group. ${ }^{11}$ Similarly, other studies using data from the National Cancer Database (NCDB) have shown no difference in decreased overall survival for patients receiving chemoradiation compared with chemotherapy alone. ${ }^{12-14}$ Furthermore, a meta-analysis demonstrated similar survival results and increased toxicity with chemoradiotherapy regimens. ${ }^{15}$ Therefore, current retrospective series do not strongly favor the use of chemoradiotherapy to improve overall survival in PDAC.

As we await prospective randomized trials evaluating neoadjuvant radiotherapy, it is important to recognize that simply demonstrating differences in survival is not enough to advance our knowledge of PDAC, and that further understanding of the biology is essential. To that end, as new trials are designed, we must strive to incorporate highquality correlative studies. Because PDAC is a heterogeneous disease, differences in outcomes in prior studies likely reflect this notion to some degree. To advance understanding of the etiology for this heterogeneity, more robust mutational analyses and correlation with behavior are necessary. Current endeavors, such as the "Know Your Tumor Initiative" from the Pancreatic Cancer Network and the COMPASS trial have used genomics-driven techniques on PDAC tumor specimens to predict chemotherapy susceptibility through biomarker identification, but substantial room exists to expand our biomarker knowledge using newer advanced techniques, such as long-read whole-genome sequencing and RNA sequencing. ${ }^{16,17}$ Long-read whole-genome sequencing has been demonstrated to improve the detection rate of variants through identification of recombination events and nucleotide repeats, whereas RNA sequencing can allow for transcriptomic analysis. ${ }^{18}$ Validating new biomarkers in this way will serve to inform surgeons and oncologists about prognosis, susceptibility, and toxicity of various therapies, among other benefits.

Ultimately, the authors have produced a study demonstrating prolonged survival using a neoadjuvant radiation with concurrent full doses of chemotherapy in the treatment of PDAC. Their study has demonstrated the feasibility of this approach, but randomized trials are needed before this treatment paradigm can be incorporated into standard-of-care practice in countries where $\mathrm{S} 1$ is available. Future studies should incorporate biomarker end points to advance our knowledge about disease biology.

\section{REFERENCES}

1. Worni M, Guller U, White RR, et al. Modest improvement in overall survival for patients with metastatic pancreatic cancer: a trend analysis using the surveillance, epidemiology, and end results registry from 1988 to 2008. Pancreas. 2013;42:1157-63.

2. Siegel RL, Miller KD, Jemal A. Cancer statistics, 2016. $C A$ Cancer J Clin. 2016;66:7-30. 
3. Gastrointestinal Tumor Study Group. Further evidence of effective adjuvant combined radiation and chemotherapy following curative resection of pancreatic cancer. Cancer. 1987;59:2006-10.

4. Klinkenbijl JH, Jeekel J, Sahmoud T, et al. Adjuvant radiotherapy and 5-fluorouracil after curative resection of cancer of the pancreas and periampullary region: phase III trial of the EORTC gastrointestinal tract cancer cooperative group. Ann Surg. 1999;230:776.

5. Eguchi H, Takeda Y, Takahashi H, et al. A prospective, openlabel multicenter phase II trial of neoadjuvant therapy using fulldose gemcitabine and S-1 concurrent with radiation for resectable pancreatic ductal adenocarcinoma. Ann of Surg Onc. 2019. In press.

6. Van Tienhoven G, Versteijne E, Suker M, et al. Preoperative chemoradiotherapy versus immediate surgery for resectable and borderline resectable pancreatic cancer (PREOPANC-1): a randomized, controlled, multicenter phase III trial (abstract). J Clin Oncol. 2018;36(Suppl 18):LBA4002-LBA4002.

7. Unno M, Motoi F, Matsuyama Y, et al. Randomized phase II/III trial of neoadjuvant chemotherapy with gemcitabine and S-1 versus upfront surgery for resectable pancreatic cancer (Prep-02/ JSAP-05) (abstract). J Clin Oncol. 2019;37(Suppl 189):4.

8. Murphy JE, Wo JY, Ryan DP, et al. Total neoadjuvant therapy with FOLFIRINOX followed by individualized chemoradiotherapy for borderline resectable pancreatic adenocarcinoma: a phase 2 clinical trial. JAMA Oncol. 2018;4:963-9.

9. Beane JD, House MG, Pitt SC, et al. Distal pancreatectomy with celiac axis resection: what are the added risks? HPB Oxf. 2015;17:777-84.

10. Katz MG, Ou F, Herman, JM, et al. Alliance for clinical trials in oncology (ALLIANCE) trial A021501: preoperative extended chemotherapy vs chemotherapy plus hypofractionated radiation therapy for borderline resectable adenocarcinoma of the head of the pancreas. BMC Cancer. 2017; 17:505.
11. Cloyd JM; Chen H, Wang X, Chemotherapy versus chemoradiation as preoperative therapy for resectable pancreatic ductal adenocarcinoma: a propensity score-adjusted analysis. Pancreas. 2019;48:216-22.

12. Mokdad, AA, Minter, RM, Yopp, AC, et al. Comparison of overall survival between preoperative chemotherapy and chemoradiotherapy for resectable pancreatic adenocarcinoma. $J$ Natl Compr Cancer Netw. 2018;16:1468-75.

13. Lutfi W, Talamonti MS, Kantor O, et al. Neoadjuvant external beam radiation is associated with no benefit in overall survival for early-stage pancreatic cancer. Am J Surg. 2017;213;521-5.

14. Franko J, Hsu JW, Thirunavukarasu, P, et al. Chemotherapy and radiation components of neoadjuvant treatment of pancreatic head adenocarcinoma: impact on perioperative mortality and long-term survival. Eur J Surg Oncol. 2017;43:351-7.

15. Liao WC, Chien KL, Lin YL, et al. Adjuvant treatments for resected pancreatic adenocarcinoma: a systematic review and network meta-analysis. Lancet Oncol. 2013;14:1095.

16. Pishvaian MJ, Bender RJ, Halverson D, et al. Molecular profiling of patients with pancreatic cancer: initial results from the Know Your Tumor Initiative. Clin Cancer Res. 2018; 24:5.

17. Aung KL, Fischer SE, Denroche RE, et al. Genomics-driven precision medicine for advanced pancreatic cancer: early results from the COMPASS trial. Clin Cancer Res. 2018;24:1344-54.

18. Nattestad M, Goodwin S, Ng K, et al. Complex rearrangements and oncogene amplifications revealed by long-read DNA and RNA sequencing of a breast cancer cell line. Genome Res. 2018;28:1126-35.

Publisher's Note Springer Nature remains neutral with regard to jurisdictional claims in published maps and institutional affiliations. 